\title{
Using Performance-Based Risk-Sharing Arrangements to Address Uncertainty in Indication-Based Pricing
}

\author{
Kai Yeung, PharmD, PhD; Meng Li, ScM; and Josh J. Carlson, PhD, MPH
}

\begin{abstract}
BACKGROUND: The rise in pharmaceutical expenditures in recent years has increased health care payer interest in ensuring good value for the money. Indication-based pricing (IBP) sets separate, indication-specific prices paid to the manufacturer according to the expected efficacy of a drug in each of its indications. IBP allows payers to consistently pay for value across indications. While promising, a limitation of IBP as originally conceived is that efficacy estimates are typically based on clinical trial data, which may differ from real-world effectiveness. An outcomes guarantee is a type of performance-based risk-sharing arrangement that adjusts payments according to prospectively tracked outcomes. We suggest that an outcomes guarantee contract, which has been used by some payers, may be adapted to achieve indication-based prices supported by real-world effectiveness.
\end{abstract}

OBJECTIVE: To illustrate the potential of an outcomes guarantee to achieve indication-based prices aligned with real-world value, using a case study of trastuzumab for the treatment of metastatic breast and advanced gastric cancers.

METHODS: We estimated costs and outcomes under traditional IBP (i.e., expected value IBP) and outcomes guarantee frameworks and calculated incremental cost-effectiveness ratios (ICERs) comparing treatment with and without trastuzumab. Efficacy data came from pivotal trials, whereas effectiveness data came from observational studies. We adjusted trastuzumab prices in order to achieve target ICERs of $\$ 150,000$ per qualityadjusted life-year under each framework and for each indication.

RESULTS: To achieve the ICER target under traditional IBP, the unit price of trastuzumab using efficacy evidence was adjusted for metastatic breast and advanced gastric cancers from an average sales price of $\$ 9.17$ per $\mathrm{mg}$ to $\$ 3.50$ per $\mathrm{mg}$ and $\$ 0.93$ per $\mathrm{mg}$, respectively. Under an outcomes guarantee, the unit price of trastuzumab using effectiveness evidence was adjusted for metastatic breast cancer and advanced gastric cancer to $\$ 8.66$ per $\mathrm{mg}$ and $\$ 0.20$ per $\mathrm{mg}$, respectively.

CONCLUSIONS: Like expected value IBP, outcomes guarantee contracts can also vary payment based on indication. In addition, an outcomes guarantee can also reduce uncertainty regarding effectiveness and better align payment with the actual value of a treatment.

J Manag Care Spec Pharm. 2017;23(10):1010-15

Copyright $\odot 2017$, Academy of Managed Care Pharmacy. All rights reserved.

\section{What is already known about this subject}

Indication-based pricing (IBP) has been proposed as a method to reimburse drug manufacturers according to indicationspecific value.

IBP draws on efficacy data from clinical trials in order to infer the expected value in each indication, but in many cases, estimates derived from efficacy data differs from effectiveness data based on the actual experiences of a health plan's patient population.

\section{What this study adds}

This study showed that performance-based risk-sharing arrangements, specifically an outcomes guarantee, can be used to address the uncertainty in real-world clinical and economic outcomes and reimburse according to indication-specific value.

A simulation of the use of trastuzumab for the treatment of metastatic breast and gastric cancers shows that relative to an outcomes guarantee contract, a traditional IBP would be likely to produce an underpayment for metastatic breast cancer and overpayment for advanced gastric cancer.

Payers and manufacturers desiring to implement a value-based purchasing scheme for pharmaceuticals should consider outcomes guarantee contracts when the real-world value is uncertain and when there are analytic resources to support such agreements.

T he rise in pharmaceutical expenditures in recent years has increased health care payer interest in ensuring good value for the money. This interest has led to a number of proposed value-based payment models. One model that has been discussed and implemented to a limited degree is indication-based pricing (IBP). ${ }^{1-3}$ IBP is based on the observation that a given drug used for multiple indications can have different levels of efficacy for each indication; therefore, at a fixed unit price (e.g., dollars per mg dose), the value achieved may differ by indication. ${ }^{1}$ IBP varies the unit price paid for each indication in order to achieve value parity across indications - an outcome consistent with the movement toward value-based health care.

A number of health care payers domestically and internationally have implemented or are planning to implement IBP in the near future. Express Scripts, the largest pharmacy benefit management (PBM) company in the United States, began implementing IBP for 3 cancers in 2016: non-small cell lung cancer, renal cancer, and prostate cancer. If successful, the program may be expanded to other oncologic and autoimmune conditions. ${ }^{4}$ Beginning in 2017, CVS Caremark, another large U.S. PBM company, plans to implement IBP for hepatitis $C$ and autoimmune conditions. ${ }^{1,5,6}$ Although the exact details are not yet known, the PBMs may use existing systems such as those used to enable prior authorizations to operationalize these programs. Outside the United States, Australia has implemented contracts in which a manufacturer is reimbursed for a single price that is the weighted-average of the expected 
use across indications multiplied by the value-based price for each indication. Italy has implemented contracts in which different levels of discounts off a single price are set for different indications. ${ }^{7,8}$ Patient registries can then be used to measure indication-specific use. ${ }^{9}$

One component of value-based payment that IBP was not designed to address is uncertainty regarding the realworld effectiveness of a treatment. ${ }^{10}$ As conceived, IBP draws on efficacy data from clinical trials in order to infer the expected value of the treatment for each indication. However, in many cases, efficacy data from clinical trials may differ from effectiveness data based on the actual experiences of a health plan's patient population because of patient heterogeneity and nonmodifiable factors such as population demographics. In these cases, alternative approaches are necessary to achieve value parity across indications that are aligned with actual patient experiences. We propose that performance-based risk-sharing arrangements, specifically outcomes guarantee contracts, are a way to implement IBP that is aligned with real-world value.

Performance-based risk sharing is traditionally viewed as a method for payers to manage clinical and economic uncertainty at the time of a drug's market entry or expansion by sharing some the financial risk associated with the uncertainty with the manufacturer.11,12 An outcomes guarantee is a particular type of risk-sharing scheme in which an outcome target and reimbursement level are agreed on at the outset, and outcomes are tracked. ${ }^{13}$ If a drug fails to meet the agreed-upon outcome target, the manufacturer provides financial adjustments (i.e., rebates, refund, or price adjustments) in order to achieve the agreed-upon reimbursement level.

In theory, the manufacturer could also receive a higher price if the drug outperforms its expectation. This approach may be adapted to match cost with benefit in an indication-specific manner. For example, if the contracted outcome measure is the same across indications, and is an accurate proxy for all the potential health effects of an intervention, an outcomes guarantee would result in different amounts of rebates or price adjustments based on the indication-specific effectiveness.

An outcomes guarantee differs from traditional IBP in that it resolves uncertainty by measuring the actual clinical and economic outcomes of patients within the payer's insured population. Hence, the net price paid at the end of the contracted period is aligned with the actual value of the product in the realworld setting. In contrast, traditional IBP does not use data from the payer's experience but uses data available before contracting, such as data from the pivotal trials at the time of drug approval. Whereas a traditional IBP may contract based on expected value, an outcomes guarantee contract is based on realized value (hereafter, traditional IBP based on expected value is referred to as "expected value IBP"). This point is illustrated by using a trastuzumab case study for the treatment of metastatic breast cancer and advanced gastric cancer.

\section{Case Study: Trastuzumab}

Trastuzumab is indicated for metastatic breast cancer and advanced gastric cancer. ${ }^{14}$ Incremental median progressionfree survival and overall survival differ across indications. ${ }^{13,15,16}$ Under these circumstances, a payer may wish to enter into an IBP arrangement, since it would obtain different levels of value at a single fixed unit price for trastuzumab. Furthermore, at the time of regulatory approval, there was uncertainty regarding the real-world effectiveness of trastuzumab, so a payer may also consider an outcomes guarantee scheme.

For this study, we compared the effect of IBP using efficacy and effectiveness data and their implications on value obtained by the payer. This comparison is illustrated by a stylized example of the use of trastuzmab in the treatment of metastatic breast cancer and advanced gastric cancer. A minimal modeling approach is used that calculates value based on mean estimates, which are then adjusted for quality of life. The intent of the calculations was not to present precise value estimates but to illustrate the different conclusions that can be drawn from different types of payer-manufacturer agreements.

\section{Methods}

\section{Analytic Overview}

IBP was simulated using expected and realized value evidence frameworks according to a U.S. perspective in which a payer and manufacturer agree on an outcomes target of $\$ 150,000$ per quality-adjusted life-year (QALY), a threshold above which interventions are generally considered low value. ${ }^{17-19}$ While the explicit use of cost-effectiveness analysis in the United States is still uncommon, there are increasing examples of such applications. ${ }^{17,18,20-22}$

Under the expected value framework, this model used efficacy evidence inputs from the pivotal randomized trials for trastuzumab. ${ }^{13,15,16}$ These inputs represent the evidence available to a payer on market entry. Under the realized value framework, the model used effectiveness evidence from observational studies. These data are a proxy for the type of data that would be generated from a payer's experience. Real-world effectiveness estimates were derived from 2 published observational cohorts: (1) a cohort of patients with newly diagnosed HER2-positive metastatic breast cancer that had not received previous systemic therapy for metastatic disease and (2) a cohort of patients with HER2-positive advanced gastric cancer who had not received previous systemic therapy for metastatic disease. ${ }^{23,24}$ The majority of patients in these 2 studies received either trastuzumab with chemotherapy or chemotherapy alone. ${ }^{23,24}$ For each of 4 scenarios-metastatic breast cancer and advanced gastric cancer under expected and realized value evidence frameworks - we calculated the incremental cost-effectiveness ratio (ICER) of using trastuzumab plus chemotherapy versus chemotherapy alone in the metastatic 


\begin{tabular}{|c|c|c|c|c|c|c|}
\hline 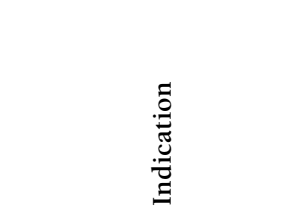 & 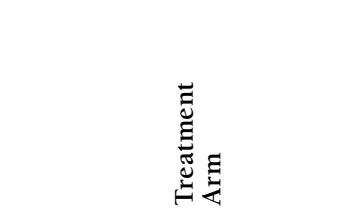 & 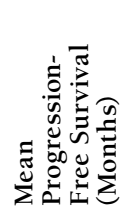 & 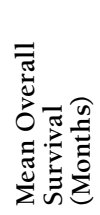 & 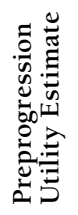 & 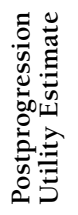 & 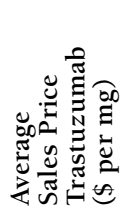 \\
\hline \multicolumn{7}{|c|}{ Parameters under an expected value indication-based price } \\
\hline \multirow{2}{*}{ Metastatic breast cancer } & Trastuzumab + chemotherapy ${ }^{a}$ & 7.5 & 25.1 & 0.8 & 0.5 & 9.17 \\
\hline & Chemotherapy alone $^{\mathrm{a}}$ & 5.0 & 20.3 & 0.8 & 0.5 & \\
\hline \multirow{2}{*}{ Advanced gastric cancer } & Trastuzumab + chemotherapyb & 6.7 & 13.8 & 0.8 & 0.6 & 9.17 \\
\hline & Chemotherapy alone $^{\mathrm{b}}$ & 5.5 & 11.1 & 0.8 & 0.6 & \\
\hline \multicolumn{7}{|c|}{ Parameters under an outcomes guarantee } \\
\hline \multirow{2}{*}{ Metastatic breast cancer } & Trastuzumab + chemotherapy ${ }^{\mathrm{a}}$ & 15.7 & 54.6 & 0.8 & 0.5 & 9.17 \\
\hline & 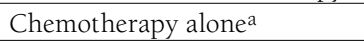 & 5.1 & 39.5 & 0.8 & 0.5 & \\
\hline \multirow{2}{*}{ Advanced gastric cancer } & Trastuzumab + chemotherapyb & 10.7 & 21.4 & 0.8 & 0.6 & 9.17 \\
\hline & Chemotherapy alone ${ }^{b}$ & 8.7 & 16.3 & 0.8 & 0.6 & \\
\hline
\end{tabular}

breast cancer setting and the advanced gastric cancer setting. We then adjusted the unit price paid in each indication to achieve the outcomes target of $\$ 150,000$ per QALY.

\section{Patient Population}

For metastatic breast cancer, individuals were modeled to have had metastatic disease that overexpressed HER2 and to not have previously received chemotherapy for their metastatic disease. For advanced gastric cancer, individuals were modeled to have had locally advanced, recurrent, or metastatic disease that overexpressed HER2 and to not have previously received chemotherapy for their metastatic disease.

\section{Health Outcomes}

The effect of trastuzumab on QALYs was modeled mainly as a function of longer progression-free survival and overall survival. The mean estimates for survival were calculated from the median survival estimates in the pivotal trials or the observational studies using an exponential function, which is a parsimonious choice for our minimal modeling approach for

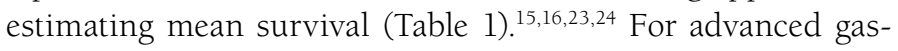
tric cancer, the preprogression utility estimates were 0.82 for trastuzumab plus chemotherapy and 0.80 for chemotherapy alone. ${ }^{25}$ The postprogression utility estimates were 0.60 for trastuzumab plus chemotherapy and 0.58 for chemotherapy alone..$^{25}$ For metastatic breast cancer, the preprogression utility estimates were 0.77 for both arms and the postprogression utility estimates were 0.49 for both arms. ${ }^{26}$

\section{Costs}

For our simplified model, the only cost inputs considered were trastuzumab acquisition cost and postprogression management costs. Trastuzumab acquisition costs was based on Medicare average sales prices from July 2016 (Table 1) ${ }^{27}$ The treatment dose was assumed to be based on prescribing information approved by the U.S. Food and Drug Administration. ${ }^{14}$ For metastatic breast cancer, treatment dosage is an initial dose of $4 \mathrm{mg}$ per $\mathrm{kg}(\mathrm{mg} / \mathrm{kg})$ followed by subsequent weekly doses of $2 \mathrm{mg} / \mathrm{kg}$. For advanced gastric cancer, treatment dosage is an initial dose of $8 \mathrm{mg} / \mathrm{kg}$ followed by subsequent doses of $6 \mathrm{mg} /$ $\mathrm{kg}$ every 3 weeks. Trastuzumab therapy is assumed to continue until progression for both indications. We assumed a weight of $75 \mathrm{~kg}$ for women and $89 \mathrm{~kg}$ for men based on recent national U.S. estimates. ${ }^{28}$ All patients in the metastatic breast cancer pivotal trial and in the observational study were women.

In the advanced gastric cancer trial, $77 \%$ of patients treated with trastuzumab were men. In the advanced gastric cancer observational study, $75 \%$ of patients treated with trastuzumab were men. Postprogression monthly costs were estimated to be $\$ 9,037$ for metastatic breast cancer and $\$ 15,280$ for advanced gastric cancer in 2015 U.S. dollars. ${ }^{29}$ Chemotherapy is recommended for 6 cycles ( 4.15 months) for all treatment arms. ${ }^{14}$ This recommendation is shorter than the mean duration in the preprogression state; therefore, the cost of chemotherapy was equivalent in all arms according to our simplified design and was not calculated.

\section{ICER and Adjusted Unit Price Calculation}

Based on the mean duration in preprogression and postprogression states, we calculated the mean costs and QALYs accrued in each state. These costs and QALYs were summed across states, and the difference in the treatment arms was used to calculate the ICER. This ICER was compared with the target ICER of $\$ 150,000$ per QALY. Using the same model, 


\begin{tabular}{|c|c|c|c|c|c|c|c|c|c|c|c|c|}
\hline & 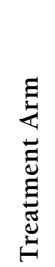 & 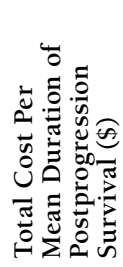 & 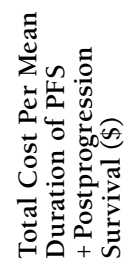 & 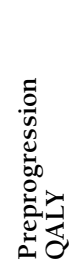 & 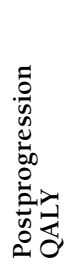 & 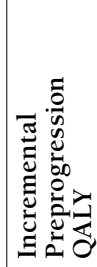 & 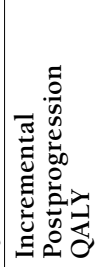 & 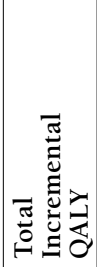 & 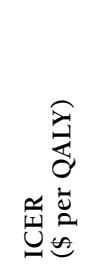 & 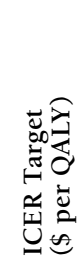 & 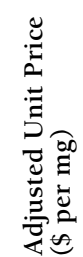 & 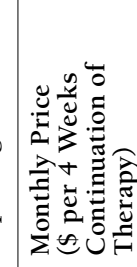 \\
\hline
\end{tabular}

Results under an expected value indication-based price

\begin{tabular}{|c|c|c|c|c|c|c|c|c|c|c|c|c|}
\hline \multirow{2}{*}{$\begin{array}{l}\text { Metastatic breast } \\
\text { cancer }\end{array}$} & Trastuzumab + chemotherapy ${ }^{a}$ & \multirow{2}{*}{$29,986.56$} & \multirow{2}{*}{$95,715.06$} & 0.7 & 1.0 & \multirow{2}{*}{0.2} & \multirow{2}{*}{0.1} & \multirow{2}{*}{0.4} & \multirow{2}{*}{260,857} & \multirow{2}{*}{150,000} & \multirow{2}{*}{3.50} & \multirow{2}{*}{$2,270.80$} \\
\hline & Chemotherapy alone $e^{a}$ & & & 0.5 & 0.9 & & & & & & & \\
\hline \multirow{2}{*}{$\begin{array}{l}\text { Advanced gastric } \\
\text { cancer }\end{array}$} & Trastuzumab + chemotherapy $b$ & \multirow{2}{*}{$33,066.57$} & \multirow{2}{*}{$81,456.10$} & 0.7 & 0.5 & \multirow{2}{*}{0.1} & \multirow{2}{*}{0.1} & \multirow{2}{*}{0.3} & \multirow{2}{*}{321,746} & \multirow{2}{*}{150,000} & \multirow{2}{*}{0.93} & \multirow{2}{*}{691.30} \\
\hline & Chemotherapy alone $^{\mathrm{b}}$ & & & 0.5 & 0.4 & & & & & & & \\
\hline \multicolumn{13}{|c|}{ Results under an outcomes guarantee } \\
\hline \multirow{2}{*}{$\begin{array}{l}\text { Metastatic breast } \\
\text { cancer }\end{array}$} & Trastuzumab + chemotherapy ${ }^{a}$ & \multirow{2}{*}{$40,193.54$} & \multirow{2}{*}{$135,128.84$} & 1.0 & 1.6 & \multirow{2}{*}{0.7} & \multirow{2}{*}{0.2} & \multirow{2}{*}{0.9} & \multirow{2}{*}{156,159} & \multirow{2}{*}{150,000} & \multirow{2}{*}{8.66} & \multirow{2}{*}{$5,623.40$} \\
\hline & Chemotherapy alone $^{\mathrm{a}}$ & & & 0.3 & 1.4 & & & & & & & \\
\hline \multirow{2}{*}{$\begin{array}{l}\text { Advanced gastric } \\
\text { cancer }\end{array}$} & Trastuzumab + chemotherapyb & \multirow{2}{*}{$46,293.20$} & \multirow{2}{*}{$99,450.62$} & 0.7 & 0.5 & \multirow{2}{*}{0.2} & \multirow{2}{*}{0.2} & \multirow{2}{*}{0.3} & \multirow{2}{*}{314,419} & 150000 & & \\
\hline & Chemotherapy alone ${ }^{b}$ & & & 0.6 & 0.4 & & & & & & 0.20 & \\
\hline
\end{tabular}

${ }^{a}$ Chemotherapy may be an anthracycline with cyclophosphamide or paclitaxel alone. ${ }^{16}$

${ }^{b}$ Chemotherapy is a fluoropyrimidine and cisplatin. ${ }^{15}$

ICER = incremental cost-effectiveness ratio; $P F S=$ progression-free survival; $Q A L Y=$ quality-adjusted life-year

we then calculated the drug unit price needed to produce the target value. That is, if the observed ICER was above the target ICER, the unit price would be adjusted downwards, and if the observed ICER was below the target ICER, the unit price would be adjusted upwards.

\section{Results}

For the metastatic breast cancer indication, trastuzumab yielded mean expected incremental QALYs gained of 0.4 at a mean expected incremental cost of $\$ 95,715$ per patient (Table 2). Therefore, the mean expected ICER was $\$ 260,857$ per QALY. In contrast, the mean observed incremental QALYs gained was 0.9 at a mean observed cost of $\$ 135,128$ per patient (Table 2). Therefore, the mean observed ICER was $\$ 156,159$ per QALY.

For the advanced gastric cancer indication, trastuzumab yielded mean expected incremental QALYs gained of 0.3 at a mean expected incremental cost of $\$ 81,456$ per patient (Table 2 ). Therefore, the mean expected ICER was $\$ 321,746$ per QALY. In contrast, the mean observed incremental QALYs gained was 0.3 at a mean observed cost of $\$ 99,450$ per patient (Table 2). Therefore, the mean observed ICER was $\$ 314,419$ per QALY.

To achieve the ICER target of $\$ 150,000$ per QALY, the unit price under the expected value IBP was adjusted downward for metastatic breast and gastric cancer from $\$ 9.17$ per mg to $\$ 3.50$ per $\mathrm{mg}$ and $\$ 0.93$ per $\mathrm{mg}$, respectively (Table 2 ). To achieve the same ICER target, the unit price under the outcomes guarantee contract was adjusted downward for metastatic breast cancer and gastric cancer to $\$ 8.66$ per $\mathrm{mg}$ and $\$ 0.20$ per $\mathrm{mg}$, respectively.

\section{Discussion}

We demonstrated that contracts using expected and realized value evidence frameworks can arrive at different value estimates and, consequently, different adjusted unit prices. In the expected value framework, the unit price would need to be lowered for metastatic breast cancer and advanced gastric cancer in order to achieve the $\$ 150,000$ per QALY threshold. In the realized value framework, the unit price would need to be lowered even further for the treatment of advanced gastric cancer and lowered much less for metastatic breast cancer. Hence, if a health plan had used an expected value IBP, it would have overpaid in gastric cancer and underpaid in breast cancer according to the actual value that was realized. This illustrative example demonstrates the potential use of outcomes guarantee contracts in implementing IBP and addressing uncertainty in real-world effectiveness.

The use of performance-based arrangements continues to receive support worldwide and increasingly so in the United States. Using the Performance Based Risk Sharing (PBRS) database at the University of Washington, we identified 66 cases of these arrangements in the United States and 6 in 2016. ${ }^{30}$ Performance-based risk sharing has been proposed as a model for state Medicaid drug benefits. ${ }^{31}$ Adoption of these arrangements by public payers would signal a paradigm shift toward realized value within the U.S. health care system. However, experiences with these arrangements have been mixed, and there remains considerable work to be done before they can and should see increased adoption. 


\section{Limitations}

While theoretically appealing, an outcomes guarantee arrangement imposes larger data collection requirements than expected value IBP. Outcomes guarantee contracts require measurement of outcomes after the fact. Such measurement and analysis may be difficult because of lack of data, expertise, or funding. ${ }^{32}$ While outcomes measurement is a challenge, there are examples of outcomes guarantee models that have overcome these barriers. ${ }^{6}$ For example, the Italian Medicines Agency has set up registries for over 40 drugs to monitor patient outcomes and to engage in risk-sharing arrangements with manufacturers. ${ }^{11}$

In some cases, such as when the outcome measured is duration on therapy, the measure is readily available in administrative claims databases. However, if clinical outcomes are the basis for outcomes guarantee models, electronic medical records or specific data collection systems will likely be needed. When selecting the outcome measures for outcomes guarantee models, payers need to strike a balance between the clinical relevance of the outcome, feasibility of data collection, and practicality because some clinically relevant outcomes may be too rare within the contract period to be the basis for an outcomes guarantee model (e.g., mortality). In these circumstances, payers may consider using surrogate markers and disease modeling to project the relationship between markers and ultimate outcomes (e.g., using tumor response as opposed to progression-free survival or overall survival in cancer).

Furthermore, we believe that outcomes guarantee contracts are not entirely a replacement for expected value IBP but suggest that they may be a way to address uncertainty in realized value and can be used to inform a more appropriate adjusted unit price. For example, a payer may consider measuring outcomes for 2 years under an outcomes guarantee contract and then, having resolved considerable uncertainty in realized value, replace the contract with IBP, along with a discount or rebate scheme. In this way, the burden of data collection would be limited to the initial contract period, and the IBP would more closely align with the plan-specific realized value.

Accurate measurement is another consideration when selecting outcomes for an outcomes guarantee model. We observed longer progression-free survival in observational studies for metastatic breast cancer patients and advanced gastric cancer patients than in pivotal trials but observed similar overall survival. One possible explanation for the observed difference is that patients are less closely monitored in observational studies than in pivotal trials, so the time of progression may be less accurately captured. The stronger the association between the clinical effectiveness measure and the comprehensive outcome of interest in terms of value (e.g., life-years or QALYs), the better the outcomes guarantee will be aligned with realized value.

Finally, it should be noted that IBP should not be perceived primarily as a method for reducing health care expenditures outright (few health care innovations do) but primarily as a method of aligning payment with value. ${ }^{33}$ Since manufacturers pursue drug development with a view to expected returns, IBP can encourage development for high value-indication populations, even if the population is relatively small. Such preferences seem to be in line with societal preferences for allocating resources for severe or life-threatening rare conditions.

\section{Conclusions}

Given the renewed interest in value-based purchasing and the specific interest in IBP, we suggest that payers and manufacturers could also consider use of outcomes guarantee contracts. As with expected value IBP, these contracts can vary payment based on indication. In addition, outcomes guarantee contracts can also reduce uncertainty regarding the real-world effectiveness of a product and better align payment with the product's actual value in the target population. Although considerable challenges remain, outcomes guarantee contracts may be considered a viable option in many settings to align prices with realized value.

\section{Authors}

KAI YEUNG, PharmD, PhD, Kaiser Permanente Washington Health Research Institute, Seattle, and Pharmaceutical Outcomes Research and Policy Program, University of Washington, Seattle. MENG LI, ScM, and JOSH J. CARLSON, PhD, MPH, Pharmaceutical Outcomes Research and Policy Program, University of Washington School of Pharmacy, Seattle.

AUTHOR CORRESPONDENCE: Kai Yeung, PharmD, PhD, Kaiser Permanente Washington Health Research Institute, 1730 Minor Ave., Ste. 1600, Seattle, WA 98101. Tel.: 206.287.2900;

E-mail: yeung.k@ghc.org.

\section{DISCLOSURES}

No funding supported this study. Carlson reports consulting fees from Genentech, Pfizer, and Seattle Genetics. The other authors have no conflicts of interest to disclose.

Study concept and design were contributed by Carlson, Yeung, and Li. Yeung Carlson, and Li collected and analyzed the data. The manuscript was written primarily by Yeung, along with Carlson and $\mathrm{Li}$, and revised by all the authors.

\section{REFERENCES}

1. Bach PB. Indication-specific pricing for cancer drugs. JAMA. 2014; 312(16):1629-30.

2. Pearson SD, Dreitlein B, Henshall C. Indication-specific pricing of pharmaceuticals in the U.S. health care system: a report from the 2015 ICER Membership Policy Summit. Institute for Clinical and Economic Review. March 2016. Available at: https://icer-review.org/wp-content/ uploads/2015/03/Final-Report-2015-ICER-Policy-Summit-on-Indicationspecific-Pricing-March-2016_revised-icons-002.pdf. Accessed August 9, 2017. 
3. Mestre-Ferrandiz J, Towse A, Dellamano R, Pistollato M. Multi-indication pricing: pros, cons and applicability to the UK. Seminiar Briefing 18. Office of Health Economics. October 2015. Available at: https://www.ohe.org/publications/multi-indication-pricing-pros-cons-and-applicability-uk. Accessed August 9, 2017.

4. Kitamura M, Koch J. Express Scripts aims new price model at cancer, inflammation. Bloomberg Business. November 16, 2015. Available at: https:// www.bloomberg.com/amp/news/articles/2015-11-16/express-scripts-to-applynew-price-model-to-auto-immune-diseases. Accessed August 9, 2017.

5. CVS Health. 2017 formulary strategy. 2016. Available at: https://cvshealth com/sites/default/files/cvs-health-2017-standard-formulary-list.pdf. Accessed August 9, 2017

6. Barlas S. Health plans and drug companies dip their toes into value-based pricing: the pressure is on P\&T committees to monitor utilization. P T. 2016;41(1):39-53

7. Australian Government Department of Health. Guidelines for Preparing Submissions to the Pharmaceutical Benefits Advisory Committee (Version 4.5). Canberra, Australia: Department of Health; 2015. Available at: https://pbac. pbs.gov.au/content/information/printable-files/pbacg-book.pdf. Accessed August 21, 2017.

8. Paris V, Belloni A. Value in pharmaceutical pricing. OECD Health Working Papers. No. 63. 2013. Paris: OECD Publishing. Available at: http:// dx.doi.org/10.1787/5k43jc9v6knx-en. Accessed August 9, 2017.

9. Agenzia Italiana del Farmaco. Lista aggiornata dei Registri e dei Piani Terapeutici. Available at: http://www.agenziafarmaco.gov.it/content/listaaggiornata-dei-registri-e-dei-piani-terapeutici-web-based. Accessed August 21, 2017.

10. Bach PB. Alternative pricing strategies for cancer drugs-reply. JAMA. 2015;313(8):858

11. Garrison LP Jr, Towse A, Briggs A, et al. Performance-based risk-sharing arrangements-good practices for design, implementation, and evaluation: report of the ISPOR good practices for performance-based risk-sharing arrangements task force. Value Health. 2013;16(5):703-19.

12. Carlson JJ, Garrison LP Jr, Sullivan SD. Paying for outcomes: innovative coverage and reimbursement schemes for pharmaceuticals. J Manag Care Pharm. 2009;15(8):683-87. Available at: http://www.jmcp.org/doi/10.18553/ jmcp.2009.15.8.683.

13. Carlson JJ, Sullivan SD, Garrison LP, Neumann PJ, Veenstra DL. Linking payment to health outcomes: a taxonomy and examination of performance-based reimbursement schemes between healthcare payers and manufacturers. Health Policy. 2010;96(3):179-90

14. HERCEPTIN (trastuzumab) intravenous infusion. Genentech. Revised March 2016. Available at: http://www.accessdata.fda.gov/drugsatfda_docs/ label/2016/103792s5330lbl.pdf. Accessed August 9, 2017.

15. Bang YJ, Van Cutsem E, Feyereislova A, et al. Trastuzumab in combination with chemotherapy versus chemotherapy alone for treatment of HER2-positive advanced gastric or gastro-oesophageal junction cancer (ToGA): a phase 3, open-label, randomised controlled trial. Lancet. 2010;376(9742):687-97.

16. Slamon DJ, Leyland-Jones B, Shak S, et al. Use of chemotherapy plus a monoclonal antibody against HER2 for metastatic breast cancer that overexpresses HER2. N Engl J Med. 2001;344(11):783-92.

17. Anderson JL, Heidenreich PA, Barnett PG, et al. ACC/AHA statement on cost/value methodology in clinical practice guidelines and performance measures: a report of the American College of Cardiology/American Heart Association Task Force on Performance Measures and Task Force on Practice Guidelines. Circulation. 2014;129(22):2329-45.
18. Institute for Clinical and Economic Review. Targeted immune modulators for rheumatoid arthritis: effectiveness \& value. Evidence report. April 7, 2017. Available at: https://icer-review.org/wp-content/uploads/2016/08/ NE_CEPAC_RA_Evidence_Report_FINAL_040717.pdf. Accessed August 9, 2017

19. World Health Organization. The World Health Report 2002: Reducing Risks, Promoting Healthy Life. Geneva: World Health Organization; 2002. Available at: http://apps.who.int/iris/bitstream/10665/42510/1/WHR_2002.pdf. Accessed August 9, 2017

20. Sullivan SD, Yeung K, Vogeler C, et al. Design, implementation, and first-year outcomes of a value-based drug formulary. J Manag Care Spec Pharm. 2015;21(4):269-75. Available at: http://www.jmcp.org/doi/10.18553/ jmcp.2015.21.4.269.

21. Yeung K, Basu A, Hansen RN, Watkins JB, Sullivan SD. Impact of a value-based formulary on medication utilization, health services utilization, and expenditures. Med Care. 2017;55(2):191-98.

22. Yeung K, Basu A, Marcum ZA, Watkins JB, Sullivan SD. Impact of a value-based formulary in three chronic disease cohorts. Am J Manag Care. 2017;23(3 Suppl):S46-S53. Available at: http://www.ajmc.com/journals/ supplement/2017/beyond-charitable-assistance-sustainable-strategies-forproviding-access-to-critical-medications/impact-of-a-value-based-formularyin-three-chronic-disease-cohorts/P-1. Accessed August 9, 2017.

23. Rugo HS, Brufsky AM, Ulcickas Yood M, et al. Racial disparities in treatment patterns and clinical outcomes in patients with HER2-positive metastatic breast cancer. Breast Cancer Res Treat. 2013;141(3):461-70.

24. Qiu MZ, Li Q, Wang ZQ, et al. HER2-positive patients receiving trastuzumab treatment have a comparable prognosis with HER2-negative advanced gastric cancer patients: a prospective cohort observation. Int J Cancer. 2014;134(10):2468-77.

25. Shiroiwa T, Fukuda T, Shimozuma K. Cost-effectiveness analysis of trastuzumab to treat HER2-positive advanced gastric cancer based on the randomised ToGA trial. Br J Cancer. 2011;105(9):1273-78.

26. Elkin EB, Weinstein MC, Winer EP, Kuntz KM, Schnitt SJ, Weeks JC. HER-2 testing and trastuzumab therapy for metastatic breast cancer: a costeffectiveness analysis. J Clin Oncol. 2004;22(5):854-63.

27. Centers for Medicare \& Medicaid Services. 2016 ASP drug pricing files. Available at: https://www.cms.gov/Medicare/Medicare-Fee-for-Service-PartB-Drugs/McrPartBDrugAvgSalesPrice/2016ASPFiles.html. Accessed August 21, 2017.

28. National Center for Health Statistics. Health, United States, 2015, with special feature on racial and ethnic health disparities. 2016. Available at: https://www.cdc.gov/nchs/data/hus/hus15.pdf. Accessed August 9, 2017.

29. Mariotto AB, Yabroff KR, Shao Y, Feuer EJ, Brown ML. Projections of the cost of cancer care in the United States: 2010-2020. J Natl Cancer Inst. 2011;103(2):117-28.

30. University of Washington School of Pharmacy. Pharmaceutical Outcomes Research \& Policy Program. Performance Based Risk Sharing (PBRS) Database. Case database. 2016. Available at: https://depts.washington. edu/pbrs/index.php. Accessed August 9, 2017.

31. Stuard S, Beyer J, Bonetto M, et al. State Medicaid alternative reimbursement and purchasing test for high-cost drugs (SMART-D): summary report. Center for Evidence-Based Policy, Oregon Health \& Science University. September 2016. Available at: http://smart-d.org/wp-content/uploads/2016/09/ SMART-D-Summary-Report-Final.pdf. Accessed August 9, 2017.

32. Drummond M. When do performance-based risk-sharing arrangements make sense? Eur J Health Econ. 2015;16(6):569-71.

33. Chandra A, Garthwaite C. The economics of indication-based drug pricing. N Engl J Med. 2017;377(2):103-06. 